RIIE (2020), Año 11 (14), 70-81.

DOI: http://dx.doi.org/10.30972/riie.11144672
Dilemas y desafios de la formación para el trabajo en la educación de jóvenes y adultos.

\title{
DILEMAS Y DESAFÍOS DE LA FORMACIÓN PARA EL TRABAJO EN LA EDUCACIÓN DE JÓVENES Y ADULTOS
}

\section{DILEMMAS AND CHALLENGES OF TRAINING FOR WORK IN YOUTH AND ADULT EDUCATION}

María Alejandra Bowman ${ }^{1}$

Fecha de recepción: 21-12-2020

Fecha de aceptación y versión final: 28-12-2020

\section{Resumen}

Este artículo presenta preocupaciones formativas y curriculares planteadas por docentes de centros de educativos de nivel primario de jóvenes y adultos, en el marco de un proceso de capacitación realizado en los años 2018 y 2019. Esta experiencia educativa se produjo por convenio institucional entre un equipo de investigación de la Universidad Nacional de Córdoba y la modalidad de educación de jóvenes y adultos de la Municipalidad de la Ciudad de Córdoba, Argentina. Se recogen específicamente en este artículo, las discusiones, reflexiones y desarrollos en torno al Área de Formación y Trabajo, producidos bajo una metodología de trabajo en taller. Se presentan tres dimensiones que pueden entenderse como dilemas que asume la formación para el trabajo como espacio curricular de nivel primario para jóvenes y adultos: 1-Dimensión teórico-conceptual sobre formación y trabajo; 2-Dimensión social y pedagógica: saberes del trabajo y trayectorias; 3-Dimensión contextual-territorial: la escuela y el barrio. Finalmente, se recuperan estas dimensiones para dejar planteados los desafíos principales del área curricular.

Palabras clave: educación - formación - jóvenes y adultos - trabajo.

\section{Abstract}

This article presents formative and curricular concerns raised by teachers of primary-level educational centers for young people and adults, within the framework of a training process carried out in 2018 and 2019. This educational experience was produced by institutional agreement between a research team of the National University of Córdoba and the modality of education for young people and adults of the Municipality of the City of Córdoba, Argentinian. The discussions, reflections and developments around the Training and Work Area, produced under a workshop methodology, are specifically collected in this article. Three dimensions are presented that can be understood as dilemmas assumed by job training as a primary level curricular space for youth and adults: 1-Theoretical-conceptual dimension on training and work; 2-Social and pedagogical dimension: knowledge of work and trajectories; 3-Contextual-territorial dimension: the school and the neighborhood. Finally, these dimensions are recovered to leave raised the main challenges of the curricular area.

Key words: education - training - youth and adults - work.

\footnotetext{
${ }^{1}$ Licenciada y Doctora en Ciencias de la Educación por la Universidad Nacional de Córdoba (UNC). Profesora Adjunta en el Departamento de Ciencias de la Educación y la Salud de la Universidad Nacional de Chilecito (UNdeC). Investigadora en la línea de educación y trabajo con jóvenes y adultos, en UNC y UndeC, Córdoba, República Argentina. Docente en Instituto Superior de Formación Docente en la provincia de Córdoba. Capacitadora de docentes y profesores no docentes. Correo electrónico: mariaalebow@gmail.com
} 


\section{Encuadre de trabajo y contextualización de la propuesta formativa}

El presente escrito recoge los desarrollos producidos en una investigación en curso y las inquietudes formativas y curriculares planteadas por docentes de centros educativos de nivel primario de jóvenes y adultos, en el marco de un trabajo colectivo realizado durante los años 2018 y 2019, entre el equipo de investigación de educación de jóvenes y adultos del Centro de Investigaciones María Saleme de Burnichon, Facultad de Filosofía y Humanidades, Universidad Nacional de Córdoba ${ }^{2}$ y la Subdirección de la Modalidad de educación de jóvenes y adultos de la Municipalidad de la Ciudad de Córdoba.

El proyecto de investigación propone analizar lo que sucede en las prácticas de los educadores, atendiendo a las políticas para la modalidad, las trayectorias laborales y formativas de los docentes y sujetos estudiantes, las realidades cotidianas locales e institucionales y los procesos de apropiación de conocimientos que se generan. (Proyecto FONCYT, PICT-2016-2081).

La investigación parte de entender que no es sólo en el ámbito escolar donde los sujetos aprenden a leer y a escribir, así como tampoco constituye éste el único espacio donde construyen conocimientos necesarios para sus estrategias de supervivencia. (Lorenzatti, 2019). Los sujetos se apropian y construyen conocimientos en los diferentes contextos en los que se desenvuelven, entre ellos, el mundo del trabajo, entendido como un espacio de aprendizaje importante para la configuración de trayectorias sociales y educativas (Bowman, 2020).

Se reconoce en el proyecto que la educación y la formación para el trabajo son derechos humanos fundamentales para el desarrollo de los sujetos jóvenes y adultos, siendo estas dimensiones vitales que solo pueden ser definidas a través del análisis de situaciones sociohistóricas concretas (Bowman, 2019).

Los docentes son conocedores de las vicisitudes de la cotidianeidad del grupo de estudiantes, hecho que les permite crear los puentes para conectar y relacionar los saberes de la vida y del trabajo con los contenidos escolares. En tal sentido, las voces de los docentes son centrales para pensar y construir la especificidad de las propuestas de enseñanza con jóvenes y adultos (Arrieta, Bowman y Delprato, 2019).

Con base en lo expuesto, se abre una línea extensionista en el proyecto de investigación que se implementa con docentes de la modalidad a través de procesos de formación docente continua, dirigida a comprender los procesos de construcción de ciudadanía de jóvenes y adultos a partir de su participación en distintas experiencias formativas, y desde allí, proponer orientaciones situadas de enseñanza.

La opción metodológica de formación es la de un taller teórico-práctico, con eje en la construcción colectiva de conocimientos, la puesta en común de problemáticas curriculares y la toma de decisiones didáctico-pedagógicas. Esto significa la posibilidad de propiciar el diálogo entre pares sobre las dificultades encontradas en el aula. Se

\footnotetext{
2 Proyecto "Prácticas educativas con jóvenes y adultos: políticas, sujetos, conocimientos". FONCYT-

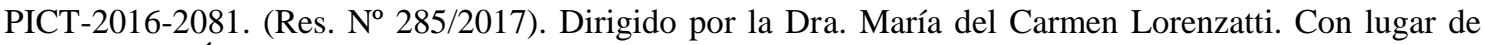
trabajo en el Área Educación del Centro de Investigaciones de la Facultad de Filosofía y Humanidades. Desde el 2017 y continúa.
} 
procura compartir las decisiones relativas a la organización de la enseñanza en el marco de las condiciones institucionales del trabajo docente: la selección de los conocimientos a enseñar; la relación de las decisiones tomadas con las características del grupo de estudiantes; las conceptualizaciones realizadas y el reconocimiento de los conocimientos de los jóvenes y adultos que se ponen en juego en cada encuentro (Lorenzatti, 2019).

Durante el año 2018 (entre marzo y noviembre), se realizaron encuentros generales con la participación de cuarenta y seis docentes de nivel primario de jóvenes y adultos, y encuentros por comisiones de trabajo que variaron en cantidad de integrantes. Se constituyeron siete comisiones de participación optativa, según el interés formativo de los docentes sobre la dimensión histórico-política de la modalidad, los sujetos y el trabajo específico con las áreas curriculares: Ciencias, Lengua, Matemática, Lenguajes Artísticos; Formación para el trabajo y proyectos comunitarios. Desde esta instancia de formación, se elaboró un documento denominado "Cuaderno de Trabajo: Notas para la Enseñanza en centros educativos de jóvenes y adultos" (2019) que se constituyó en un insumo básico de trabajo en el año subsiguiente.

Durante el año 2019 (entre marzo y junio) se dio continuidad al proceso formativo, asumiendo como foco la elaboración de núcleos conceptuales a partir de problemáticas sociales comunitarias relevadas por las escuelas.

Los núcleos conceptuales se tratan de un modo de organización integrada de los contenidos a enseñar en una estructura que permite abordar, discutir y profundizar una problemática desde distintas áreas curriculares, cuestionar el por qué, el para qué de los hechos o fenómenos y relacionar los procesos inmediatos y mediatos. La potencialidad de los mismos radica en la red conceptual que construyen los docentes en función de los intereses, los saberes del mundo social y las condiciones de vida de los estudiantes (Lorenzatti, 2019; Arrieta, Bowman \& Delprato, 2019).

Este proceso nos condujo a analizar los problemas barriales-comunitarios que inciden en las trayectorias sociales y educativas de los estudiantes, y en las condiciones institucionales de las escuelas municipales, y -desde allí- recoger las principales preocupaciones de los docentes para definir una problemática social y delinear criterios de selección de contenidos integrando diversas áreas curriculares.

En esta instancia, se combinó el trabajo en taller en dos encuentros a grupo total (apertura y cierre); con dos encuentros en agrupamientos de docentes por zonas de la ciudad (norte, noroeste, sur, sureste), respetando la cercanía y la organización cotidiana del trabajo entre pares (desarrollo conjunto de proyectos comunitarios, de propuestas artísticas, entre otras).

En este artículo, se plantean -puntualmente- las preocupaciones en torno a la enseñanza que surgieron en los tres talleres de la comisión del Área de Formación para el Trabajo conformada por siete docentes, en 2018; y los planteos que emergieron en los cuatro talleres de formación desarrollados en 2019.

Este escrito se estructura en base a tres dimensiones que pueden entenderse como dilemas que presenta la formación para el trabajo como espacio curricular de nivel primario con jóvenes y adultos: 1-Dimensión teórico-conceptual sobre formación y trabajo; 2-Dimensión social y pedagógica: saberes del trabajo y trayectorias; 3 Dimensión contextual-territorial: la escuela y el barrio. Finalmente, se recuperan estos 
dilemas para dejar planteados los desafíos principales del área curricular, en el marco de una formación integral para jóvenes y adultos.

\section{Dimensión teórico-conceptual sobre formación y trabajo}

Muchos de los planteos de los docentes surgen de la necesidad de diferenciar el concepto de trabajo como noción ampliada, en relación con el de empleo como un término restringido. En esta línea, se expuso la dificultad de abordar la problemática del trabajo con grupos tan heterogéneos en condiciones socioeconómicas, en intereses y en expectativas.

Los estudiantes se caracterizan por ser adultos mayores jubilados que trabajan desde temprana edad, jóvenes desocupados que no buscan trabajo, jóvenes y adultos con trabajos precarios y/o esporádicos, jóvenes y adultos que subsisten por ayudas sociales estatales, entre otras. Las mujeres en general son trabajadoras en casas particulares no registradas, quienes son madres sostienen casi exclusivamente la vida cotidiana de su hogar y familia.

La mayoría de los estudiantes adultos mayores, no pudieron realizar la escuela primaria debido a que trabajaban en el campo con sus padres, y se encontraban a mucha distancia de una institución educativa. En muchos casos, a sus familias no les pagaban, sino que era sólo un intercambio de productos por su trabajo, lo que hacía que los hijos debieran salir a trabajar desde temprana edad para contribuir a la manutención familiar (Lorenzatti, 2019, p.43).

En el marco de esta heterogeneidad de trayectorias laborales y sociales de los jóvenes y adultos, una de las tensiones principales que se desprenden del trabajo realizado junto a docentes giran en torno a dos interrogantes: ¿qué significa formar para el trabajo? ¿de qué conceptualización de trabajo partimos?

La vinculación entre educación y trabajo es una preocupación histórica que se ha dado desde los orígenes de nuestro sistema educativo nacional. La incorporación de contenidos del mundo del trabajo a la enseñanza fue uno de los ejes principales en torno al cual giraron los debates en la educación de jóvenes y adultos, a lo largo del siglo XX. Este vínculo histórico no ha estado exento de polémicas y contradicciones. Los saberes laborales se han transmitido de diversas maneras a lo largo de la historia, generalmente asociados con la educación técnica, asumiendo un lugar subordinado (Bowman, 2019).

Actualmente, la formación para el trabajo es un núcleo central de la educación, es uno de los ejes centrales propuestos por la política educativa vigente, a partir de la Ley de Educación Técnico Profesional (26.058) del año 2005, y de la Ley de Educación Nacional (26.206) del año 2006.

A partir de las normativas particulares sobre la educación de jóvenes y adultos se crearon áreas curriculares específicas sobre Formación para el trabajo en las jurisdicciones, y diversos "dispositivos de acercamiento" al mundo del trabajo como el desarrollo de pasantías educativas y estrategias organizativas que promueven convenios colaborativos entre diferentes instituciones orientados a fortalecer los vínculos con los sectores socio-productivos locales donde están insertas las escuelas 
(Bowman, 2019). En este sentido, se sostiene que el enfoque de que la formación para el trabajo concierne sólo a la escuela técnica se ha ido superando (Jacinto, 2013).

En la Propuesta Curricular para Alfabetización y Nivel Primario de Adultos (2008) de la provincia de Córdoba, aprobada por la Resolución 378/08, se incorporó el Área de Formación para el Trabajo, como un espacio para abordar contenidos sobre el mundo del trabajo y como un lugar desde donde desarrollar la articulación con las organizaciones del contexto sociocultural donde está inserta la institución.

Uno de los dilemas principales que se presenta en el Área de Formación para el Trabajo es asociar indistintamente el mundo del trabajo al empleo. En este sentido, formar para el trabajo implica asumir posicionamientos teóricos y establecer algunos señalamientos sobre el sentido común que atraviesa la temática.

El trabajo como construcción social y como característica distintiva de la condición humana no es sinónimo de empleo, y adquiere múltiples formas según el contexto histórico, social y cultural.

Desde una concepción antropológica, Rieznik (2001) sostiene que el trabajo es un atributo específico de la acción del hombre dirigida a asegurar y crear las condiciones de su propia vida de un modo único y que le es propio. Es la capacidad humana de transformar la naturaleza, la posibilidad del hombre de adecuar especialmente el entorno a sus necesidades, es la condición de su misma supervivencia.

Al trabajar, el hombre desempeña frente a la naturaleza, el papel de un poder natural, pone en acción las fuerzas de que está dotado su cuerpo, brazos y piernas, cabeza y manos, a fin de asimilarse las materias dándoles una forma útil para su vida. Al mismo tiempo que, mediante este proceso, actúa sobre la naturaleza exterior y la transforma, transforma también su propia naturaleza desarrollando las propias facultades que en ella dormitan. (Karl Marx citado en Rieznik, 2001, p.9)

En esta línea, la dimensión "productiva" del trabajo no necesariamente está ligada a un salario sino a los aspectos integrales de la persona, vinculados a una definición amplia de la condición de ciudadano, a la participación en la producción y reproducción de los aspectos políticos, sociales y culturales de la vida; es lo que hacemos, y no está condicionado a la existencia de un empleador como en la "forma empleo" (Levy, 2010).

De acuerdo con estas definiciones, todos los hombres trabajan en algún sentido, cualquiera sea su situación, en términos de las categorías sociales mediante las cuales se analiza el trabajo comúnmente: empleado, desempleado, población económicamente activa, inactiva, ocupado, sub-ocupado, desocupado, precarizado. El trabajo es estructurante en la vida de las personas, es parte vital de lo que somos. Está tan asociado a la identidad y a la pertenencia social, que la persona que está "sin trabajo" pone en cuestión su propio ser (Messina, Pieck y Castañeda, 2008).

En este sentido, formar para el mundo del trabajo, no es sólo abordar el empleo como actividad remunerada sujeta a una relación contractual, sino también la capacidad de generar un abanico de actividades más amplias: cooperativas, solidarias, voluntarias, recreativas, participativas, educativas, entre otras. Desde esta 
conceptualización sobre el trabajo, entendemos que la educación es una herramienta fundamental para la formación integral del ciudadano.

Formar para el trabajo en espacios escolares con jóvenes y adultos, entonces, no es educar para un puesto laboral específico, sino que asume un sentido más amplio. El objetivo principal es formar una conducta colectiva donde el sujeto sea agente activo, es decir, educar sujetos capaces de desenvolverse en el medio laboral con sensibilidad y conciencia social sobre los problemas que los afectan como base para su transformación.

En torno a lo expuesto, la formación para el trabajo tiene un alto contenido social, y la selección de contenidos escolares, su jerarquización y las mediaciones pedagógico-didácticas construidas por los docentes son centrales para propiciar un espacio de discusión y de aprendizaje significativo anclado a las historias de vida de los sujetos jóvenes y adultos.

En este marco, el conocimiento sobre las transformaciones producidas en el mundo del trabajo en las últimas décadas, es un aspecto central que los docentes deben considerar para la formación porque se trata de procesos que también atraviesan e interpelan las condiciones de vida propias en tanto educadores, trabajadores y ciudadanos.

\section{Dimensión social pedagógica: saberes del trabajo y trayectorias}

Una problemática en torno a la formación para el trabajo que se desprende del dilema formativo anteriormente expuesto, se trata de cómo entramar los trayectos de vida de los jóvenes y adultos, sus diversos recorridos por el mundo laboral y sus saberes adquiridos en distintos ámbitos de participación social a los contenidos escolares. Se trata de otro eje central de discusión para pensar propuestas formativas de forma situada.

Recuperar las trayectorias sociales y laborales, es uno de los propósitos centrales del Área de Formación para el trabajo en el nivel primario para jóvenes y adultos en la provincia de Córdoba. En el marco del proceso formativo con docentes se recogieron dos interrogantes que plantean este segundo dilema: ¿qué conocemos de las trayectorias sociales y laborales de nuestros estudiantes jóvenes y adultos? ¿qué saberes sobre el trabajo reconocemos y recuperamos los docentes en el proceso de enseñanza?

Para el sistema educativo argentino que se organiza en el siglo XIX, los conocimientos básicos sobre el mundo del trabajo ocuparon un "lugar marginal" en el tiempo dedicado respecto a los saberes académicos. Tenían más valor los saberes vinculados a lo racional y la alta cultura letrada que los saberes del mundo del trabajo, populares y prácticos (Pineau, 2003; Dussel y Southwell, 2012).

Un pedagogo pionero en plantear la necesidad de vincular los conocimientos de la vida cotidiana de los sujetos jóvenes y adultos con los contenidos escolares, fue Paulo Freire, quien manifiesta que, en el contexto concreto de los sujetos, las relaciones entre práctica y saber de la práctica son inseparables:

Es imposible que enseñemos contenidos sin saber cómo piensan los alumnos en su contexto real, en su vida cotidiana; sin saber lo que ellos saben 
independientemente de la escuela, para ayudarlos, por un lado, a saber, mejor lo que ya saben, y por el otro lado para enseñarles, a partir de ahí, lo que aún no saben. No podemos dejar de considerar las condiciones materiales desfavorables que experimentan muchos alumnos de las escuelas de la periferia de la ciudad, lo precario de sus viviendas, las deficiencias de su alimentación, la falta de actividades de lectura de la palabra en su vida cotidiana, de estudio escolar, su convivencia con la violencia y con la muerte, de la que casi siempre se vuelven íntimos. (Freire, 2002, p.129)

En un plano más específico, sobre los saberes del trabajo que construyen jóvenes y adultos, Spinosa (2006) manifiesta que es necesario capitalizar en los espacios formativos la experiencia de trabajo en la medida que constituye, en sí misma, un espacio de aprendizaje. Para ello es preciso definir las características de los saberes que se construyen en el trabajo, su vinculación con la experiencia escolar y las formas posibles de articulación.

Según el autor, todo "saber hacer" supone un concepto (implícito o explícito), y lo mismo ocurre con los saberes de situación ("saber qué hacer"). El reconocimiento de la existencia, producción y circulación de este tipo de saberes en el trabajo, resulta trascendente ya que en sí mismo cuestiona la idea difundida, que la experiencia solo genera saberes de experiencia.

La experiencia no es solo la rutinización de actos. Inclusive en aquellas actividades que resultan más repetitivas y monótonas, no es posible pensar en la acumulación de experiencia sin tener en cuenta la reflexión que cada sujeto realiza sobre la misma. El problema es que muchas veces esta reflexión no llega a formularse en términos explícitos. En el acto de trabajo existe siempre, en la medida que se resuelven problemas, un análisis de la actividad, un diagnóstico que orienta la acción posterior. No obstante, en la experiencia y en las condiciones cotidianas definidas para el trabajo, este proceso de diagnóstico y de acción se efectúa sin mediación de la palabra (Spinosa, 2006).

La consideración de los saberes del trabajo, permite la organización de trayectos escolares que posibilitan el aprovechamiento de la experiencia social de vida de las personas jóvenes y adultas, como parte de un aprendizaje que no reemplaza a la formación sistemática del sistema educativo, pero a la que puede integrarse.

Es importante y necesario reconocer los saberes del trabajo en el empleo "informal", ya que en la experiencia se construyen saberes aun cuando no tienen la "formalidad" que la escolaridad requiere, aun cuando los propios sujetos jóvenes y adultos no lo reconozcan como saberes.

El abordaje de las trayectorias sociales y laborales como puente para la enseñanza es una estrategia para la comprensión de historias adversas desde donde proponer un espacio formativo que posibilite re-significar y desnaturalizar prácticas desiguales del mundo del trabajo. Asimismo, permite que el estudiante y el docente reconozcan capacidades, saberes y habilidades adquiridas en diferentes ámbitos de participación en su vida cotidiana.

La diversidad de saberes, circunstancias y singularidades de los recorridos de vida legitima los aprendizajes con los otros, constituyendo esto una fortaleza para la educación. En este sentido, se parte de una perspectiva de educación permanente 
que revaloriza las propias experiencias y los trabajos de la vida cotidiana de los estudiantes jóvenes y adultos (Lorenzatti, 2019, p.106).

La reflexión sobre la práctica cotidiana es pensada en relación estrecha con la experiencia de vida y de trabajo que los jóvenes y adultos adquieren en sus trayectorias sociales y laborales, y puede constituirse en una valiosa herramienta de aprendizaje que merece ser considerada en los espacios de formación a partir de la creación de situaciones didácticas que permitan la confrontación de los esquemas de conocimiento propios de cada sujeto con el funcionamiento del mundo social, con el propósito de permitir la transformación del pensamiento en formas cada vez más complejas y abstractas.

\section{Dimensión contextual- territorial: la escuela y el barrio}

Los procesos de globalización, los profundos cambios en las tecnologías de la comunicación y los transportes, la internacionalización y concentración de la riqueza, reforzaron las tendencias al desarrollo desigual de los pueblos latinoamericanos. Esto ha profundizado los problemas que se presentan para la formación para el trabajo, la dificultad de responder a sectores atravesados por múltiples desigualdades sociales, con una población empleada de forma precaria, con alta informalidad, cuentapropismo, en condiciones extremas de autosubsistencia (Bowman, 2020).

La mayoría de los estudiantes jóvenes y adultos asistentes a escuelas municipales habita en asentamientos, villas y barrios pobres de la periferia de la ciudad de Córdoba Capital, muchos de ellos sobreviven a través de la recolección de basura en las calles, "cartoneando", limpiando casas particulares u otros lugares ajenos. Otra característica distintiva de las escuelas primarias de jóvenes y adultos del ámbito municipal de Córdoba, es el desarrollo de proyectos comunitarios que abordan problemáticas sociales de los barrios donde se insertan las escuelas.

El Proyecto Comunitario se implementa a partir de "redes comunitarias" en el territorio en articulación con distintos organismos locales, dependencias estatales y organizaciones no gubernamentales. Está orientado a favorecer la organización colectiva y la formación para el trabajo, mediante la estructuración de vínculos entre grupos con preocupaciones y necesidades comunes. Los adultos participan activamente en los espacios comunitarios de su barrio o contexto local, especialmente en los centros vecinales o centros de jubilados.

Un nudo temático que surge en el proceso desarrollado junto a docentes es cómo se entrama la escuela a las problemáticas del contexto local. Desde la articulación del proyecto comunitario a la formación para el trabajo, se plantean interrogantes que traducen el tercer dilema: ¿cómo tender puentes desde la escuela para potenciar el vínculo con la comunidad local? ¿qué problemáticas comunitarias priorizar para articular conocimientos escolares y cotidianos? ¿qué lugar ocupa la escuela en contextos locales atravesados por la desigualdad social y qué valor asume la formación para el trabajo y el proyecto comunitario como instrumentos políticos de transformación social?

Este dilema surge sobre la discusión en torno al reconocimiento de las preocupaciones y problemáticas barriales que atraviesan los estudiantes: pobreza, desempleo, precariedad laboral, contaminación ambiental, falta de servicios básicos, entre otras. En este sentido, se propone una estrategia diferenciada que tenga en 
cuenta la especificidad de los problemas y las necesidades educativas en un marco local comunitario.

Tender puentes con el contexto local de las escuelas desde el Proyecto Comunitario, ayuda a promover la organización colectiva y producir articulaciones con instituciones de formación profesional y/o capacitación laboral, con centros de salud, con centros vecinales, con propuestas artísticas y recreativas, entre otras. Uno de los condicionamientos principales que puede producirse es la falta de implicancia de las instituciones locales con la escuela, y que sea el docente quien resuelva -de forma individual- la necesidad de diseñar ofertas de capacitación laboral (autoempleo, oficios, emprendimientos, etc.) y/o de ofrecer ciertos conocimientos prácticos que no poseen en la base de su formación.

Según Krichesky (2006) la relación de la escuela con la comunidad cumple un importante rol en el desarrollo de propuestas y alternativas de acción orientadas a promover procesos de inclusión educativa. La posibilidad de que otros actores sociales (familias, vecinos, organizaciones, asociaciones, clubes, etc.) formen parte de la vida escolar constituye en muchos casos un punto de partida para que la gestión institucional y la propuesta pedagógica resulte más democrática.

En las normativas vigentes de la modalidad de educación de jóvenes y adultos, se reafirma el derecho a que se valoren y reconozcan los saberes adquiridos fuera del sistema educativo y la necesidad de una formación integral a través de un curriculum flexible. Se reconoce a la escuela como un lugar privilegiado para la elaboración y desarrollo de proyectos que permitan instalar el futuro como posibilidad, el conocimiento, la creatividad y la responsabilidad como herramientas para transformar la realidad.

La escuela es el territorio de lo público, de lo común, de lo diverso. Es el espacio donde se entraman historias de vida, desafíos, promesas y utopías, también trayectorias dramáticas marcadas por la desigualdad y la exclusión. El territorio de la escuela es mucho más que su edificio compartido por turnos y niveles, es el ámbito donde se construyen conocimientos, donde se gestan lazos profundos con los niños, jóvenes y adultos de la comunidad local.

Es fundamental, entonces, resaltar que la orientación de la enseñanza y de los proyectos comunitarios debe ser la "solidaridad" y que su rasgo principal se define también por ser un lugar de mediación en la construcción del mismo sujeto social (Cullen, 1997).

\section{Desafíos nodales de la formación para el trabajo}

Para finalizar este escrito, se recogen los principales dilemas señalados en el cuerpo del artículo para dejar planteados -a modo de conclusión- los desafíos nodales de la temática.

Los desafíos para la formación para el trabajo son grandes y complejos. Al educar para el trabajo también se educa para la vida. Esta responsabilidad educativa debe ser entendida como un valor social, político, ético, como un compromiso inexorable con aquellos jóvenes y adultos que atraviesan mayores desventajas sociales. 
Asumir el sentido social del trabajo desde una concepción amplia, como capacidad creadora y de transformación, implica interactuar de manera solidaria con el medio social en el que se vive, movilizar y ejercitar derechos, identificarse con los problemas que aquejan al barrio y proponer soluciones. Esta perspectiva abona la posibilidad de vincular la acción social y laboral con actividades culturales y recreativas, a fin de lograr una mejor calidad de vida para los demás miembros de su comunidad y para sí mismos.

Abordar las trayectorias sociales y laborales de los jóvenes y adultos, supone reconocer y recuperar lo que los sujetos saben sobre el mundo del trabajo, sus estrategias de supervivencia, sus dificultades, sus expectativas, sus conocimientos prácticos. Este es un punto de partida para pensar las necesidades educativas y, desde allí, construir conocimientos escolares significativos anclados a la vida cotidiana de los sujetos, seleccionando el contenido en articulación con otras áreas curriculares de forma integral e integrada.

La diversidad de saberes, circunstancias y singularidades de los recorridos de vida constituye una fortaleza para la formación para el trabajo. En este sentido, es importante partir de una perspectiva educativa que revalorice las experiencias prácticas: el "saber hacer", el "saber qué hacer" (Spinosa, 2006) y que visibilice las diversas modalidades que asume el trabajo en los estudiantes jóvenes y adultos.

La escuela necesita pensar y comprender las problemáticas locales para intervenir pedagógicamente. Integrar la escuela al territorio, y el territorio a la escuela, supone la creación de estrategias formativas que hagan de estos ámbitos un lugar donde alojarse, acompañarse y producir nuevos conocimientos.

La implicación del colectivo escolar en las distintas problemáticas del barrio es clave en el objetivo de participación y organización social. En el marco de la formación para el trabajo, los proyectos comunitarios pueden funcionar como articuladores de programas de empleo y capacitaciones laborales que se implementan desde otras instituciones en el espacio local. También pueden ser una vía para demandar al Estado el cumplimiento de sus obligaciones y la producción de formas propias de resolución de necesidades básicas tales como: la salud, el empleo, el transporte, los espacios de recreación, la promoción de derechos, entre otros.

La educación de jóvenes y adultos cobra un sentido político y social vital en las tramas que teje con el contexto local, en la capacidad de generar lazos afectivos y colaborativos, en los intercambios que recrean un horizonte común de transformación, en los compromisos asumidos que fortalecen la diversidad, la convergencia, la negociación, la labor conjunta.

El compromiso de muchos equipos docentes en el desarrollo de experiencias cooperativas y participativas, desafían las rigideces de la organización escolar. No obstante, son espacios que no se logran de una vez y para siempre, sino que necesitan sostenerse en un esfuerzo continuo.

Es necesario partir de un concepto ampliado de trabajo, reconocer y valorar las trayectorias y saberes de los estudiantes y sus familias, articular instancias de acción con la comunidad local para poder identificar con claridad cuáles son los proyectos posibles y viables- que permiten crear circuitos de formación integral a jóvenes y adultos como sujetos de derecho, que se orientan a una mayor inclusión social. 
El barrio y la comunidad local se potencian con ideas y acciones situadas, con experiencias educativas innovadoras, con la construcción de nuevas formas de hacer escuela, aun cuando las propuestas de formación presentan limitaciones (de recursos, de espacios, de tiempos), aun cuando parecieran quedar invisibilizadas por la envergadura y la complejidad de las problemáticas abordadas. Existen muchas iniciativas de esta naturaleza en las escuelas municipales de adultos, están ahí, tan vivas, tan necesarias y urgentes.

\section{Referencias bibliográficas}

Arrieta, R., Bowman, M. A. y Delprato, M. F. (2019). Formación docente y enseñanza en Educación de Jóvenes y Adultos. Análisis de una experiencia de trabajo colaborativo con docentes municipales. En Actas del $X$ Encuentro Interdisciplinario de Ciencias Humanas y Sociales, Facultad de Filosofía y Humanidades, UNC. 27, 28 y 29 de noviembre de 2019, Córdoba, Argentina. Recuperado de https://ffyh.unc.edu.ar/publicaciones/wpcontent/uploads/sites/35/2020/10/ActasXEncuentroTomo1.pdf

Bowman, M.A (2019). La formación para el trabajo en la educación de jóvenes y adultos. Notas conceptuales y definiciones político curriculares. En M. del C. Lorenzatti y M. A. Bowman (Ed.) Educación de jóvenes y adultos. Contribuciones de la investigación para pensar la práctica educativa (pp. 101127). Río Cuarto, Córdoba, Argentina: UNIRIO.

Bowman, M. A (2020). Jóvenes, Educación y Trabajo. Políticas públicas y tramas locales de la formación laboral para jóvenes con escolaridad básica inconclusa. Río Cuarto, Córdoba, Argentina: UNIRIO. Recuperado de http://www.unirioeditora.com.ar/producto/jovenes-educacion-trabajo/

Cullen, C. (1997). Crítica de las razones de educar: temas de filosofía de la educación. Buenos Aires, Argentina: Paidós.

Dussel, I. y Southwell, M. (2012). Saberes y trabajos: miradas sobre una relación compleja. El Monitor de la educación. Trabajo y Escuela. Dossier: La relación entre trabajo y educación, 5ta. época, (29) 25-28. Recuperado de http://www.bnm.me.gov.ar/giga1/monitor/monitor/monitor 2012 n29.pdf

Dussel, I. y Pineau, P. (1995). De cuando la clase obrera entró al paraíso: la educación técnica estatal en el primer peronismo. En A. Puiggrós (Dir.). Discursos pedagógicos e imaginario social en el peronismo (1945-1955) (pp.107-176). Buenos Aires, Argentina: Galerna.

Freire, P. (2002). Pedagogía de la esperanza: un reencuentro con la pedagogía del oprimido. México: Siglo XXI.

Jacinto, C. (2013). La formación para el trabajo en la escuela secundaria como reflexión crítica y como recurso. Propuesta Educativa, Año 22, 2 (40), 48-63. Recuperado de http://propuestaeducativa.flacso.org.ar/wp-content/uploads/2019/12/40dossier-jacinto.pdf

Krichesky, M. (2006). Escuela y comunidad: desafíos para la inclusión educativa. Buenos Aires, Argentina: Ministerio de Educación, Ciencia y Tecnología de la Nación. Recuperado de http://www.bnm.me.gov.ar/giga1/documentos/EL005385.pdf 
Levy, E. (2010). Educación, trabajo y políticas sociales en Argentina. En J.A. Castorina y V. Orce (Coord.). Diálogos y reflexiones en investigación: contribuciones al campo educativo. Buenos Aires, Argentina: Editorial de la Facultad de Filosofía y Letras, Universidad de Buenos Aires.

Lorenzatti, M del C. y Bowman, M.A. (Comp.) (2019). Educación de jóvenes y adultos. Contribuciones de la investigación para pensar la práctica educativa. Río Cuarto, Córdoba, Argentina: UNIRIO. Recuperado de http://www.unirioeditora.com.ar/wp-content/uploads/2019/09/978-987-688-359-7.pdf

Lorenzatti, M. del C. (2019) (Coord.). Cuaderno de trabajo: notas para la enseñanza en Centros Educativos de Jóvenes y Adultos. Córdoba, Argentina: Editorial de la Municipalidad de Córdoba, Dirección de Patrimonio Cultural. Recuperado de https://educacion.cordoba.gob.ar/wp-content/uploads/sites/28/2019/06/cuaderno-detrabajo-notas-para-la-enseanza-en-centros-educativos-de-jvenes-y-adultos.pdf

Messina, G., Pieck, E. y Castañeda, E. (2008). Educación y trabajo: una mirada a la práctica en sectores marginados de América Latina. Santiago de Chile: OREALC/UNESCO.

Pineau, P. (2003). La vergüenza de haber sido y el dolor de ya no ser: los avatares de la educación técnica entre 1955 y 1983. En A. Puiggrós, A. (Dir.). Dictaduras y utopías en la historia reciente de la educación argentina (1955-1983), Serie Historia de la Educación en la Argentina, Volumen VIII. Bs. As., Argentina: Galerna.

Rieznik, P. (2001). Trabajo, una definición antropológica. Dossier: Trabajo, alienación y crisis en el mundo contemporáneo. En Razón y Revolución (7), 1-21. Reedición electrónica. Recuperado de https://www.razonyrevolucion.org/textos/revryr/prodetrab/ryr7Rieznik.pdf

Spinosa, M. (2006). Los saberes y el trabajo. Anales de la Educación Común, Tercer siglo, Año 2 (5), 1-11. Recuperado de http://servicios.abc.gov.ar/lainstitucion/revistacomponents/revista/archivos/anales/numer o05/archivosparaimprimir/19.spinosa.pdf 